\title{
Aspectos morfológicos de Dicheilonema rheae Owen 1843 en ñandúes (Rhea americana)
}

\author{
Comolli, J.; Santa Cruz, A.; Roux, J.; González, A. \\ Servicio de Producciones No Tradicionales, Facultad de Ciencias Veterinarias, Universidad Nacional del \\ Nordeste, Sargento Cabral 2139, Corrientes (3400), Argentina. Tel.-Fax: + 543783425753. \\ E-mail: jacomolli@vet.unne.edu.ar.
}

\begin{abstract}
Resumen
Comolli, J.; Santa Cruz, A.; Roux, J.; González, A.: Aspectos morfológicos de Dicheilonema rheae Owen 1843 en ñandúes (Rhea americana). Rev. vet. 22: 2, 141-143, 2011. El objetivo del trabajo fue reportar aspectos morfológicos del nematodo Dicheilonema rheae Owen 1843, documentados por microscopía óptica y electrónica, constituyendo la primera cita en Argentina para este parásito. Los especímenes de D. rheae $(\mathrm{n}=37)$ fueron colectados de 4 ñandúes provenientes de la Provincia de Buenos Aires, tres de ellos silvestres y uno de criadero. Los helmintos fueron conservados en solución de Railliet \& Henry, medidos y microfotografiados. Los parásitos revelaron marcado dimorfismo sexual: los machos $(n=20)$ midieron $18,24 \pm 3,61 \mathrm{~cm}$ y las hembras $(\mathrm{n}=17) 66,24 \pm 16,40 \mathrm{~cm}$. Los machos mostraron una extremidad posterior curvada ventralmente, con alas caudales bien desarrolladas y papilas alargadas con espículas desiguales. La vulva de las hembras estaba ubicada en la región esofágica. La abertura oral se encontró rodeada por espesamientos cuticulares. Debajo de la boca se encontraron cuatro pares de papilas circulares. Los huevos, de cáscara gruesa, se presentaron larvados.
\end{abstract}

Palabras clave: Dicheilonema rheae, primera cita, Argentina, Rhea americana.

\begin{abstract}
Comolli, J.; Santa Cruz, A.; Roux, J.; González, A.: Morphological aspects of Dicheilonema rheae Owen 1843 in "ñandúes" (Rhea americana). Rev. vet. 22: 2, 141-143, 2011. The objective of the trial was to report morphological aspects of the nematode Dicheilonema rheae Owen 1843, documented by optic and electronic microscopy, constituting the first reference for this parasite in Argentina. Specimens of $D$. rheae $(n=37)$ were collected from 4 "ñandúes" coming from the Buenos Aires Province, three of them wild animals and one from an hatchery. Helminths, conserved in Railliet \& Henry solution, were measured and photographied. Parasites revealed marked sexual dimorphism: males $(\mathrm{n}=20)$ measured $18.24 \pm 3.61 \mathrm{~cm}$ and females $(\mathrm{n}=17) 66.24 \pm 16.40 \mathrm{~cm}$. Males showed a caudal extremity ventrally curved, with well developed wings flows, lengthened papillas and unequal spicules. The vulva of females was located near the esophagus. The oral opening was surrounded by a cuticle thickening. Under the mouth four couples of circular papillas were present. Eggs were thick-shelled and larvated.
\end{abstract}

Key words: Dicheilonema rheae, first reference, Argentina, Rhea americana.

\section{INTRODUCCIÓN}

El ñandú (Rhea americana) es un ratite integrante de la avifauna silvestre de Argentina, que por sus características etológicas, fisiológicas y anatómicas surge como una nueva opción en las producciones no tradicionales ${ }^{3}$. Su cría en cautiverio se presenta como una alternativa en la industria de la carne, por ser considerada como la proteína del segundo milenio, con-

Recibido: 21 julio 2011 / Aceptado: 31 agosto 2011

Presentado en Reunión SGCYT-UNNE 2003 y en

XXIV Sesión Comunicaciones FCV-UNNE 2003. sumida, producida y promocionada en los países desarrollados ${ }^{4}$. Es la más grande de las aves autóctonas de Sudamérica y su área de distribución, en la Argentina, comprende las regiones centro, norte y este ${ }^{5}$. Su cría en cautiverio es problemática por provenir del ambiente silvestre y no estar acostumbrados a la reclusión. Actualmente se está promocionando su uso sustentable como recurso natural ${ }^{5,9}$.

Al ser una especie que se encuentra en proceso de domesticación resulta necesario abordar el estudio sistemático de las diversas patologías que la afectan. Entre ellas, las enfermedades parasitarias adquieren suma importancia durante el cautiverio, por el impacto nega- 
tivo que producen en la rentabilidad ${ }^{12}$. Son escasos los datos concernientes a las parasitosis de los ñandúes en cautiverio $^{2,12}$.

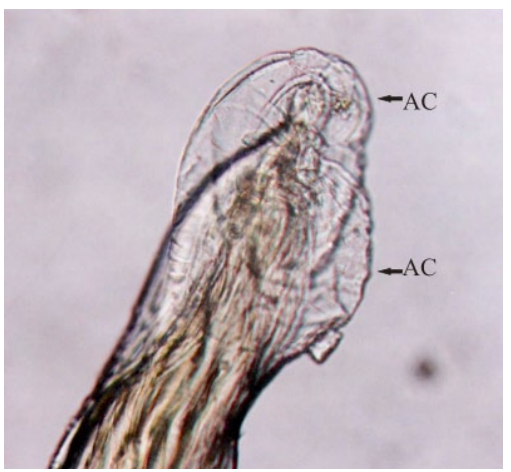

Figura 1. D. rheae. Macho, extremidad caudal. AC: alas caudales. ML (10x).

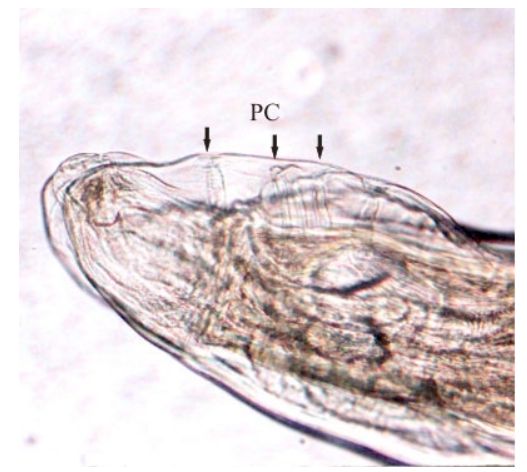

Figura 2. D. rheae. Macho, extremidad caudal. PC: papilas caudales. ML (10x).

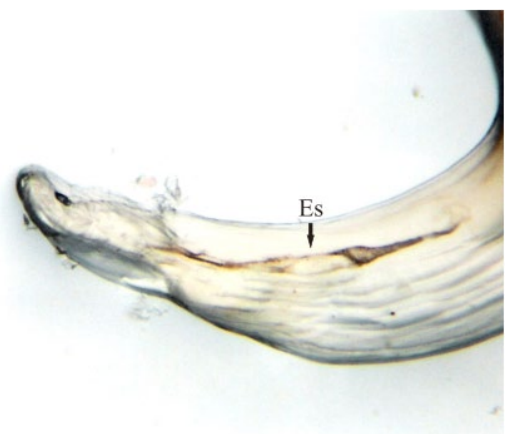

Figura 3. D. rheae. Macho, extremidad caudal. Es: espículas. ML (4x).

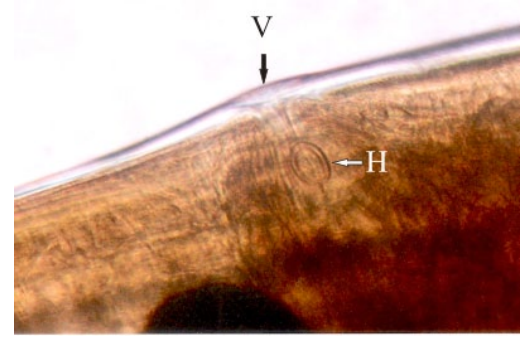

Figura 4. D. rheae. Hembra, extremidad anterior. V: vulva; H: huevo en oviducto. ML (10x).
La helmintofauna que afecta a $R$. americana es variada, unos de los nematodos más comúnmente encontrados es Dicheilonema rheae ${ }^{12}$. Este parásito se

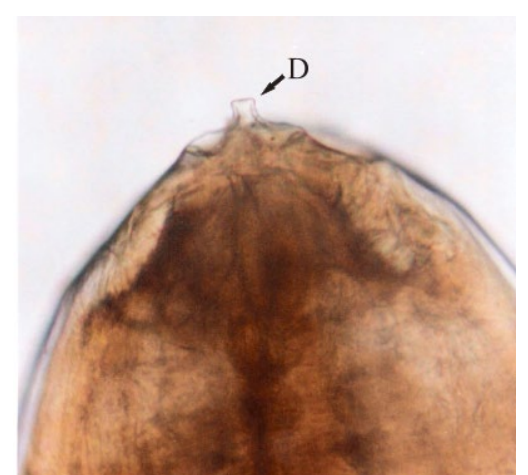

Figura 5. D. rheae. Hembra, extremidad anterior. D: Espesamientos cuticulares (“dientes"). ML (10x).



Figura 6. D. rheae. Hembra, extremidad anterior. D: espesamientos cuticulares. Ch: "charreteras". MEB (500x).

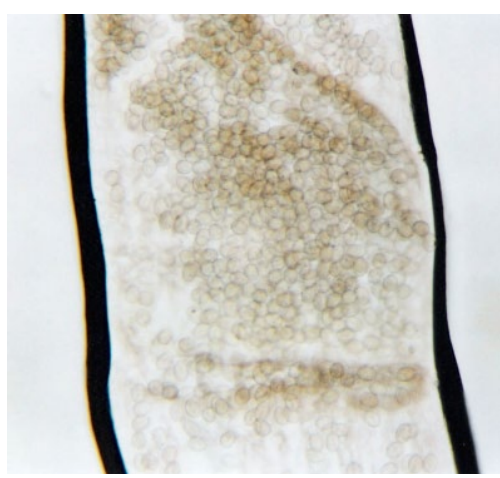

Figura 7. D. rheae. Hembra. Útero grávido. ML (4x).

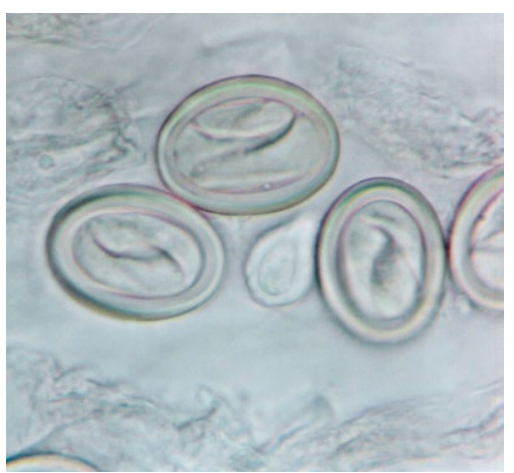

Figura 8. D. rheae. Huevos larvados ML (40x). 
catalogó inicialmente dentro de la superfamilia Filaroidea, familia Filariidae. Posteriormente se estableció su actual situación sistemática, dentro del orden Spirurida, superfamilia Diplotriaenoidea, familia Diplotriaenidae y subfamilia Dicheilonematinae 1, 7,8 . Esta clasificación se basó fundamentalmente en su ciclo biológico, en el cual las hembras oviponen huevos de cáscara gruesa con el primer estadio larval en su interior, los que al ser eliminados por el sistema respiratorio y la materia fecal son ingeridos por los artrópodos (Insecta, Acrididae y Tettigonidae) ${ }^{8}$, que en algunas ocasiones pueden ser predados por otros de mayor tamaño, los cuales participarían en el ciclo como huéspedes paraténicos ${ }^{7}$. Este nematodo fue excluido de la superfamilia Filaroidea porque la misma está restringida a las especies que producen microfilarias.

El objetivo del presente trabajo fue reportar el primer hallazgo de $D$. rheae en Argentina, así como describir sus principales características morfológicas.

\section{MATERIAL Y MÉTODOS}

Los especímenes de D. rheae fueron colectados de 4 ñandúes de diferentes sexos y edades, provenientes del sur de la Provincia de Buenos Aires, Argentina (región templada). Tres de ellos procedían del medio silvestres y uno de criadero. Los nematodes adultos fueron obtenidos de los sacos aéreos abdominales, torácicos, cavidad celómica y uno específicamente en el tejido subcutáneo de la pata izquierda (articulación femoro-tibial). Fueron conservados en solución de Railliet \& Henry y ulteriormente estudiados bajo microscopio de luz (ML) y fotografiados. Los ejemplares destinados a microscopía electrónica de barrido (MEB) se fijaron en glutaraldehído al 3\%, posteriormente se sometieron a deshidratación en acetonas de graduación creciente, secado a punto crítico, montaje, metalizado con oro-paladio, observación y registro fotográfico en un aparato JEOL $5800 \mathrm{LV}$ del Servicio de MEB de la Universidad Nacional del Nordeste (Corrientes, Argentina). Para la identificación específica de los helmintos se utilizaron claves convencionales 1,6,10,11.

\section{RESULTADOS Y DISCUSIÓN}

La observación macroscópica de los helmintos reveló que poseen un marcado dimorfismo sexual: los machos $(\mathrm{n}=20)$ presentaron una longitud media de $18,24 \pm 3,61 \mathrm{~cm}$ y las hembras $(\mathrm{n}=17)$ midieron $66,24 \pm 16,40 \mathrm{~cm}$. Los machos presentaron la extremidad posterior curvada ventralmente, alas caudales bien desarrolladas y ensambladas anteriormente, con papilas caudales alargadas (Figuras 1 y 2), espículas marcadamente diferentes (Figura 3), la izquierda dos a tres veces más larga que la derecha, de forma muy compleja. En las hembras, se observó la vulva ubicada en la región esofágica (Figura 4) y el ano atrofiado cerca del extremo posterior redondeado.

La extremidad cefálica presentó la abertura oral rodeada por elevaciones o espesamiento cuticulares.
Estas formaciones fueron descritas como semejantes a dientes (Figuras 5 y 6); por debajo de la abertura oral se encontraron cuatro pares de papilas circulares que sobresalían levemente, conocidas como "charreteras" 1 (Figura 6).

Los huevos se apreciaron dentro del útero y oviducto (Figuras 4 y 7), caracterizándose por ser elípticos y medir $20,95 \pm 0,63 \mu \mathrm{m}$ de largo y $14,95 \pm 0,93 \mu \mathrm{m}$ de ancho, con una cáscara muy gruesa, conteniendo el primer estadio larval en su interior (Figura 8), lo cual corrobora lo apreciado por autores que clasificaron a $D$. rheae dentro de la Superfamilia Diplotriaenoidea por no producir microfilarias ${ }^{7,8}$.

\section{REFERENCIAS}

1. Anderson RC, Bain O. 1976. Keys to genera of the order Spirurida. Part 3. Diplotriaenoidea, Aproctoidea and Filaroidea. In: CIH keys to the nematode parasites of vertebrates (Anderson RC, Ed.), CAB International, London, p. 59-116.

2. Comolli J, Cayo D, Santa Cruz A, Milano F, Roux J. 2006. Aspectos morfológicos de Paradeletrocephalus minor (Molin, 1861) Freitas \& Lent, 1947, en ñandú (Rhea americana), por medio de microscopio de luz y microscopio electrónico de barrido. Parasitol Latinoam 61: 183-187.

3. Dignani V, Obschatko ES. 2003. Panorama de las producciones agroalimentarias no tradicionales en la Argentina, Publ. CEPAL-ONU, Buenos Aires, p. 33-69.

4. Giannoni ML. 1996. Emas e Avestruzes, uma alternativa para o produtor rural, Ed. Funep, Jaboticabal, p. 49.

5. Martella MB, Navarro JL. 2006. Proyecto ñandú, manejo de Rhea americana y $R$. pennata en la Argentina. En: Manejo de fauna silvestre en la Argentina (Bolkovic M, Ramadori D, Ed.), Publ Secret Ambient Desarr Sustent, Buenos Aires, p. 39-50.

6. Masi Pallares R. 1990. Nematodes de los vertebrados de Paraguay y otros parásitos del nuevo mundo, Ed. Efacim, Asunción (Paraguay), p. 689.

7. Vakarenco EG. 1994. On the occurrence of paratenic parasitism in nematodes of the suborder Filariata. Sourcebestnik Zoologii 94: 678-680.

8. Vakarenco EG, Kuzmin Y. 2000. The development of Dicheilonema rheae (Nematoda: Diplotriaenoidea) in the intermediate host. Helminthol 37: 47-51.

9. Vieites C, González O. 2007. Análisis de producciones animales alternativas con potencial de desarrollo mediato e inmediato en la Argentina, Ed. Tres Tiempos, Buenos Aires, p. 211.

10. Yamaguti S. 1961. Systema helminthum Vol. III, The nematodes of vertebrates, Interscience Publisher, New York, p. 679 y 692.

11. Yorke W, Maplestone PA. 1926. The nematodes parasites of vertebrates, Ed. J\&A Churchill, London, p. 536.

12. Zettermann CD, Nascimento AA, Tebaldi JÁ, Szabó MJ. 2005. Observations on helminth infections of freeliving and captive rheas (Rhea americana) in Brazil. Vet Parasitol 129: 169-172. 